\title{
IdeAs
}

Idées d'Amériques

$6 \mid 2015$

Migrer dans les Amériques

\section{De Oaxaca à Los Angeles. Rencontre avec Odilia Romero, militante indienne en Californie}

From Oaxaca to Los Angeles. A meeting with Odilia Romero, militant indigenous people in California

De Oaxaca a Los Angeles. Encuentro con Odilia Romero, militante indígena en California

\section{Amandine DeBruyker}

\section{OpenEdition}

\section{Journals}

Édition électronique

URL : https://journals.openedition.org/ideas/1197

DOI : $10.4000 /$ ideas. 1197

ISSN : 1950-5701

Éditeur

Institut des Amériques

Référence électronique

Amandine DeBruyker, « De Oaxaca à Los Angeles. Rencontre avec Odilia Romero, militante indienne en Californie ", IdeAs [En ligne], 6 | 2015, mis en ligne le 10 décembre 2015, consulté le 20 octobre 2022. URL : http://journals.openedition.org/ideas/1197 ; DOI : https://doi.org/10.4000/ideas.1197

Ce document a été généré automatiquement le 20 octobre 2022.

\section{c)}

Creative Commons - Attribution - Pas d'Utilisation Commerciale - Pas de Modification 4.0 International - CC BY-NC-ND 4.0

https://creativecommons.org/licenses/by-nc-nd/4.0/ 


\section{De Oaxaca à Los Angeles. Rencontre avec Odilia Romero, militante indienne en Californie}

From Oaxaca to Los Angeles. A meeting with Odilia Romero, militant indigenous people in California

De Oaxaca a Los Angeles. Encuentro con Odilia Romero, militante indígena en California

Amandine DeBruyker

\section{NOTE DE L'AUTEUR}

Pour faciliter la lecture de cet entretien, un travail de réécriture a dû être opéré tout en tâchant de rester au plus près des propos de l'interviewée. L'entretien, réalisé en espagnol, comporte de nombreux anglicismes qu'il a également fallu adapter pour des raisons de compréhension/clarté.

Entretien réalisé et traduit par Amandine DeBruyker

Odilia Romero est une figure du militantisme pour la défense des droits des communautés indiennes en Californie. Née dans le village zapotèque de San Bartolomé Zoogocho, au cœur de la Sierra Norte de Juarez de l'État de Oaxaca au Mexique, elle vit à Los Angeles depuis plus de trente ans mais se considère toujours et avant tout comme indienne zoogochense. Membre actif du Front Indien d'Organisations Binationales (FIOB) ${ }^{1}$, plus communément appelé le « Front », elle est en même temps respectée pour son engagement militant et critiquée pour ses propos allant parfois au-delà du politiquement correct.

Lors de l'entretien qu'elle nous a accordé en juillet 2015, les récits de son expérience au sein de cette organisation -l'une des plus (re)connues de la région- 
ouvrent une fenêtre sur des thématiques récurrentes de son quotidien telles que la migration des Amérindiens du Mexique aux États-Unis, la discrimination et les inégalités de genre.

OdiLIA, Vous ÊTES LA VICE-COORDINATRICE gÉNÉRALE BINATIONALE DU FRONT INDIEN d'organisations binationales. pouvez-vous me parler un peu de cette ORgANISATION, DE QUOI S'OCCUPE-T-ELLE ? QUELLES SONT SES ACTIVITÉS ?

Le Front est une organisation binationale avec des bureaux et avec une base d'approximativement 5000 membres, entre ceux qui vivent dans l'Isthme de Oaxaca, les Vallées Centrales de Oaxaca, la région Mixtèque, la Basse Californie (San Quintin, Tijuana, Ensenada), et ceux qui vivent en Californie : San Diego, Los Angeles, Santa Maria et Fresno et récemment Oxnard. C'est une organisation dédiée à la défense et à la lutte pour les droits des communautés amérindiennes du Mexique, aussi bien sur les plans linguistique et culturel que sur celui du droit du travail.

Nous avons différents programmes pour les communautés migrantes. Ici et là-bas. À Oaxaca, nous faisons beaucoup de projets comme... le projet qui s'appelle le « Droit de ne pas Migrer ». C'est un projet qui tente de prévenir la migration... bon, à une échelle minime parce qu'on ne peut pas prévenir quand il n'y a pas la possibilité de survivre au Mexique mais cela se fait au travers de projets productifs comme la production de tomates, de poulets, de poulardes, et aussi comme la production de huipiles $^{2}$, de blouses [chemisiers brodés] et d'autres tissus... Ça se fait à travers ça, tenter de créer du travail et donc créer quelque chose pour que les gens puissent survivre. Et là-bas nous avons aussi les caisses d'épargne dans lesquelles on enseigne aux communautés comment investir l'argent des remesas ${ }^{3}$ qu'elles reçoivent. Donc nous avons quatorze caisses d'épargne dont les collègues adhérentes au projet sont les bailleresses, il n'y a qu'un seul homme.

Dans ce projet, elles prêtent l'argent dans la communauté pour les projets productifs $^{4}$. Si elles veulent... je ne sais pas... planter des tomates, elles demandent l'argent à la caisse, la caisse leur prête et elles remboursent avec un intérêt. Mais elles aussi sont membres de la caisse et leur argent, elles se le prêtent entre elles mais ensuite cela génère un intérêt dont les petits bénéfices sont divisés à la fin de l'année.

ET ICI IL Y A AUSSI dES PROgRAMMES À LOS ANgELES, OU EN CALIFORNIE ?

En Californie nous avons commencé avec ce qui était avant le Fond FIOB et maintenant c'est le Crédit Entrepreneur. On a pensé à ce modèle de prêt parce que les gens n'avaient pas accès à... comment ça s'appelle... aux banques où personne ne voulait leur prêter ou alors on leur faisait payer beaucoup dans les communautés, comme ils allaient chez des usuriers ou ceux qui te donnent... à un "PayCheck ${ }^{5}$ » et tout, comme ils te demandent ton salaire comme avance et ils te le donnent... et cela ne plaisait pas aux gens. Donc avec le Fond FIOB ce projet de micro-crédit a été créé, parmi les premiers ici aux États-Unis ou en Californie, et maintenant nous avons des bureaux à Santa Maria, Oxnard, Los Angeles, et nous prêtons au niveau de la Californie aux communautés qui n'ont pas accès à l'argent. Mais cet argent ce sont des investisseurs d'un groupe qui s'appelle Ayúdate (« Aide-toi ») qui nous le prêtent. Donc ce n'est pas l'argent de la communauté comme c'est le cas pour l'argent des caisses d'épargnes. C'est la différence. À Oaxaca l'argent est le leur, ici nous demandons un prêt pour pouvoir prêter. C'est de l'argent mexicain, c'est une 
association civile qui s'appelle Ayúdate qui nous prête l'argent. Donc eux ils nous prêtent l'argent à nous et nous, nous le prêtons ici aux migrants.

\section{COMMENT EN ÊTES-VOUS ARRIVÉE À TRAVAILLER AVEC LE FIOB ?}

Moi j'étais... Mes parents avaient un restaurant, et là venait un ami qui s'appelle... Bon, je ne vais pas dire son nom parce que ce n'est plus mon ami [Rires]. Ce jeune homme qui venait manger là m'a demandé si je voulais travailler pour l'organisation « sœur » du Front qui s'appelle le Centre Binational pour le Développement Indien de Oaxaca parce qu'il y avait un travail à temps partiel pour faire des ateliers sur... des salons de la santé pour la communauté : comment aller aux cliniques mobiles pour faire des tests de Papanicolaou ${ }^{6}$ ou des mammographies et tout cela. Je lui ai dit oui, parce que je n'aime pas la restauration, je n'aimais pas ça. Et là, après ça j'ai appris qu'il y avait une création du Front et moi j'étais déjà fan du Front, mais ça ne m'a jamais intéressée d'aller chercher de ce côté là parce qu'il n'y avait pas de bureau ici. Quand j'ai commencé à travailler avec l'organisation sœur du Front, j'ai dit «Bon, pourquoi nous ne réorganisons pas un Front à Los Angeles ?». Et on m'a donné la charge de réorganiser le Front à Los Angeles, et voilà. Je crois que cela fait treize ans que nous avons réhabilité le Front ici à Los Angeles. Donc depuis lors j'ai été un membre du Front et je crois qu'au bout de deux ans je suis devenue la coordinatrice des affaires féminines du Front. Au niveau binational. Et ce poste je l'ai occupé pendant deux ans... non, deux fois et chaque mandat dure trois ans, donc je l'ai occupé pendant 6 ans. Et à travers ce projet, nous avons mis en place le MIEL, Leadership des Femmes Indiennes (Mujeres Indigenas En Liderazgo), qui est un projet d'éducation et d'empowerment ${ }^{7}$ des femmes indiennes mais l'empowerment dans leurs communautés à partir de la cosmovision indienne, mais aussi l'empowerment hors de leurs communautés, et dans un cadre politique hors de ce qui est de la communauté, et donc comment naviguer entre les deux mondes en termes féminins. Nous avons commencé à traiter de la santé reproductive, qu'ils ont encore aujourd'hui à Oaxaca, les ateliers sur l'égalité de genre, la santé sexuelle et reproductive, et l'empowerment de la femme. Cela a été fait à Los Angeles, à San Diego, et une partie à Tijuana. Les seuls endroits où ça ne s'est pas fait c'est le nord de la Californie où ça n'a pas pu être mis en place.

\section{POURQUOI CELA ?}

Eh bien, il y a toujours une résistance au changement, non? Quand tu dis empowerment, il y a toujours des gens qui résistent... qu'une femme soit émancipée, ce n'est déjà pas évident, mais qu'une femme indienne soit émancipée... elle se «corrompt » comme ils disent, c'est comme... de celles qui causent des problèmes dans la communauté, donc il y a résistance. Le Front est vraiment unique parce qu'il y a des traditionalistes du mouvement indien, il y a des radicaux, mais il y a aussi les super modérés et les conservateurs... Donc dans le Front nous sommes de toutes les couleurs, nous sommes des chilaquiles ${ }^{8}$ !

\section{IL N'Y A DONC PAS UN MODÈLE DU RÔLE DE LA FEMME DANS LES COMMUNAUTÉS ET AU SEIN DU FRONT ?}

Eh bien, il faut rappeler que le Front a été créé par des hommes, que des hommes. Les femmes ont participé oui, elles prenaient des notes, elles faisaient le café, oui elles étaient là, mais dans l'histoire du Front il ne s'est que peu de fois écrit quelque chose sur la participation des femmes. C'est à dire... Et c'est la même chose dont nous parlons avec les associations, les hometown associations ou associations de oriundos ${ }^{9}$, on 
montre toujours l'homme mais la femme aussi était là: quelqu'un a fait le café, quelqu'un a été la secrétaire, quelqu'un leur a fait à manger... Et donc c'est sur ce modèle que s'est créé le Front. Mais il y a eu des femmes depuis le début.

\section{CE FUt difficile POUR VOUS d'ACCÉdER AU POSTE À RESPONSABILITÉS QUE VOUS OCCUPEZ ACTUELLEMENT AU SEIN DU FRONT ?}

Eh bien oui, c'est une lutte constante parce que si tu fais, alors tu roules certaines personnes, et si tu ne fais pas : «les femmes ne veulent pas avancer» ou «elles ne veulent pas travailler ». Il y a toujours des plaintes, non? Que tu fasses ou pas il y a toujours des critiques.

Je crois, comme ils disent, que j'ai la peau très dure donc ça ne m'offense pas qu'on m'insulte et «ok... c'est bien ». Non, mais oui c'est difficile parce qu'il y a une partie qui dit qu'il faut faire cela de la santé reproductive, il faut faire ça, il faut permettre l'empowerment... et de fait nous avons une étude qui a été réalisée par le Front luimême en son sein. Et là ça te montre qu'en Californie c'est un Front qui parle beaucoup d'égalité de genre, mais dans la pratique, c'est terrible la situation à ce sujet à l'intérieur de l'organisation. Mais à Oaxaca, là où le discours est plus moyen... Ils disent «il vaut mieux que tu ne parles pas » mais dans la pratique c'est tout le contraire : la femme peut avancer beaucoup plus. De fait j'ai été nommée comme coordinatrice générale de la part de Oaxaca mais Californie n'a pas voulu. Alors Californie dit «bon on la nomme pour ce qui est de la coordination mais pas la coordination générale ", et maintenant ils disent " bon, ils ne font rien ». Mais dépose une réclamation à ton chef ! Comme ça, c'est simple. Mais si c'était ainsi... Le Front est compliqué.

DONC IL Y A PLUS de difficultés En CALIFORNIE QU'À OAXACA POUR LES FEMMES, POUR ALLER DE L'AVANT DANS L'ORgANISATION ? POUR ACCÉdER À DES POSTES IMPORTANTS ?

Je crois que des deux côtés parce que même si Oaxaca est beaucoup plus participatif au sujet du rôle de la femme et des ateliers là-bas dans les communautés, mais dans le Front, comment dire, nous avons vingt-trois ans et il n'y a jamais eu une femme comme coordinatrice générale. Et bon, cela te donne à penser qu'en vingt-trois ans... combien y a-t-il déjà eu d'élections sans qu'il y ait de femme, et je ne crois pas que cela arrive à la prochaine élection, ni dans les deux suivantes. Moi je pense que ça va être difficile. Il existe encore beaucoup de résistance de la part des membres, des femmes comme des hommes, pour qu'une femme y arrive.

Certains disent que les femmes ne prennent pas d'initiative, qu'elles ne veulent pas des postes et qu'ensuite elles ne font rien, mais ils oublient que si tu es mère, si tu es épouse, $t u$ as beaucoup plus de désavantages que les hommes, tu dois t'occuper des enfants, et faire plein de choses... Bien sûr moi je n'ai pas ce problème parce que j'ai un compagnon qui me soutient et qui s'occupe du petit et vice versa, non? Mais... toutes les femmes n'ont pas un compagnon qui puisse les soutenir dans leur travail. Donc je pense qu'ils oublient ça et c'est facile pour eux de dire «bon, elles ne veulent pas, elles n'ont pas l'envie, elles n'aiment pas le Front... ». Mais il y a beaucoup de choses, oui. L'autre jour, certaines des femmes entre elles disaient qu'avec ce qu'elles avaient à faire... nous étions dans un atelier et elles se sont excusées, elles m'ont dit « Non, désolée d'arriver en retard mais ce qu'il se passe c'est que mon mari voulait que je mange avec lui, et je lui ai dit que non parce que je devais venir à l'atelier et il m'a dit "eh bien tu n'as qu'à aller vivre là-bas, puisque tu vas d'atelier en atelier !" ". Donc les femmes ont beaucoup plus de difficultés que les hommes qui vont et 
viennent facilement, et donc... Ce n'est pas une question du Front, je crois que c'est une question sociale et structurelle qui limite la femme et si la femme se rebelle, alors c'est une mauvaise femme ou elle apporte des problèmes, et donc...

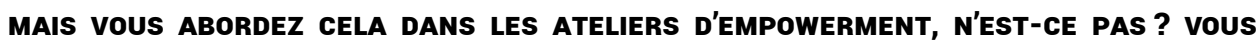
AVEZ PU OBSERVER DES CHANgEMENTS DURANT TOUT CE TEMPS AU SEIN DU FRONT ?

Oui, oui nous avons vu des changements, il y a eu par exemple Doña Mari. Elle travaillait comme femme de ménage, employée de maison et elle dit que maintenant elle est heureuse au Front parce qu'elle a appris beaucoup de choses et maintenant, bon, c'est difficile pour elle... Ce n'est pas difficile avec son mari mais c'est comme un réveil, elle avait toujours voulu travailler dans un bureau et maintenant elle travaille dans un bureau. Et elle anime les ateliers et soutient d'autres femmes, et elle dit que c'est comme... être au Front a été pour elle comme une libération. Donc il y a d'autres femmes qui ont participé, les femmes à Oaxaca disent « Non, bon, moi j'attends juste que mon époux s'en aille au Nord pour venir aux ateliers ». Donc les choses changent. Mais pour ce qui est du Front à Oaxaca, le FIOB Oaxaca c'est beaucoup de femmes parce que les hommes sont là [au Nord], et donc elles ont beaucoup plus de pouvoir de participation que les femmes d'ici parce que les maris sont ici.

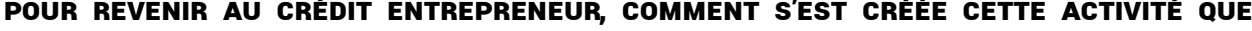
VOUS MENEZ AUJOURD'HUI ?

Eh bien simplement, une chose au Front c'est que nous sommes volontaires. Personne ne reçoit de salaire au Front et on finit par mettre de notre poche, par exemple... si tu vas à une conférence, la conférence te paie le billet mais ce que tu manges, cela vient de ta poche, et donc il n'y a pas de salaire dans le Front, nous sommes tous volontaires. Et l'une des choses que nous voulions était qu'au moins la direction puisse avoir de l'argent pour survivre pour les deux ou trois ans au Front, les deux ou trois ans en poste, que nous ayons un peu d'argent pour pouvoir dire « voilà pour ta nourriture, voilà pour ton hôtel » parce que des fois tu finis par payer toi-même l'hôtel. Et nous avons cherché comment faire cela. Pour cela, ce monsieur qui s'appelle David Velasco est venu, c'est le représentant de Ayúdate qui est une organisation mexicaine qui travaille dans le café, avec les communautés indiennes, dans ce qui est des micro-crédits et avec de nombreuses années d'expérience. Il nous a proposé si nous voulions en faire partie, j'étais alors la coordinatrice des affaires féminines et Gaspar [Rivera-Salgado ${ }^{10}$ ] était le coordinateur général et lui avait déjà parlé avec les coordinateurs généraux d'avant et ils lui avaient dit que non, que c'était très risqué et que donc non, non, non... Alors nous nous avons dit « Bon, c'est une bonne idée, ça va être très facile, nous allons entrer là-dedans ", et nous avons intégré cela mais nous n'avions jamais pensé... et bien, aujourd'hui encore nous n'avons pas l'argent pour le projet que nous avions, celui d'avoir de l'argent pour pouvoir voyager, pour faire... Le but était d'aller aux Nations Unies avec ce projet qui fournirait l'argent pour aller là-bas un mois, mais cela ne peut pas encore se faire parce que c'est un projet très coûteux. Cela nous a coûté beaucoup pour nous qui sommes dedans, beaucoup de temps... l'apprentissage... plus qu'à eux, non? Parce qu'ils ont perdu de l'argent... Ils n'ont pas perdu d'argent mais ils n'ont fait aucun bénéfice avec leur argent du fait qu'on l'a prêté aux communautés mais ils ont apporté un modèle ici à Los Angeles, et comme nous nous n'avions pas d'expérience, nous avons suivi leur modèle et peu à peu il a été modifié pour arriver à ce qu'il est aujourd'hui. Et maintenant donc, nous prêtons et récupérons presque à cent pour 
cent. Mais... Nous avons eu des problèmes avec le projet parce qu'il y a eu un moment où nous ne récupérions pas parce que les gens ne payaient pas, parce que c'était des groupes comme dans les micro-crédits qui sont populaires, c'était un groupe de cinq, un groupe de dix, et une personne était responsable et parfois celui qui était responsable gardait l'argent ou ne payait pas, ou les autres le sortaient du groupe et considéraient que ce n'était pas leur affaire, ils ne nous remettaient pas l'argent... On a donc eu beaucoup de problèmes avec le projet. Et ensuite, et bien, nous ne voulions plus prêter beaucoup, nous avons commencé à modifier et nous sommes dans une nouvelle étape du projet dans laquelle nous avons l'argent maintenant pour prêter mais nous manquons de clients. Donc nous n'avons jamais été dans la situation dans laquelle il y a des clients, de l'argent, et des paiements en même temps, la récupération. Chaque fois que nous récupérons, nous n'avons pas de clients, ou nous récupérons super bien et nous n'avons pas d'argent. Donc les trois choses qui sont importantes pour le projet n'ont jamais coïncidé. Actuellement nous sommes làdedans mais l'objectif du projet était cela, que nous ayons un accès financier, pour aller à l'Organisation des Nations Unies, et aller à tous les différents congrès/salons internationaux sur les questions de migrations, la migration indienne, mais ça ne s'est pas fait, nous espérons toujours dans les deux prochaines années... [Rires].

\section{ET CELA, C'EST VOTRE ACTIVITÉ PRINCIPALE ?}

Oui, mon principal travail... Ce travail me prend beaucoup de temps parce qu'il faut voir les chiffres, qui paie, qui ne paie pas, pourquoi ils ne paient pas, qu'est-ce qu'il se passe, et répondre aux investisseurs, à Ayúdate : Qu'est-ce qu'il se passe ? Pourquoi cette personne n'est pas venue ? Et donc c'est constant, constant. Et je crois-moi que c'est une partie du problème de pourquoi nous n'avons pas de clients parce que c'est un programme lié au Front, quand on est dans la rue, dans les réunions, dans les lieux, tu ramènes des clients parce que tu dis aux gens « c'est un projet du Front » et comme ça tu ramènes des clients. Mais si tu es tous les jours dans les chiffres, dans les chiffres et rendant des comptes, et que tu vas de réunion en réunion, et une autre réunion, et encore une sur la même chose et la même chose, eh bien tu passes beaucoup de temps dans ton bureau et tu n'es pas là où tu dois être, là où sont les gens. Je crois que ça c'est l'autre chose qui nous est arrivée. Que dans la structure que nous avons créée, nous passons beaucoup de temps en réunions et à vérifier les chiffres. Tous les jours, on demande "Où sont-ils ? Où sont-ils ? Et combien ? Qu'estce qu'il manque? Qui manque? ", je crois qu'avant le modèle que nous avions était de travailler beaucoup à la promotion et à la fin du mois nous faisions les comptes.

\section{COMBIEN D'ARgENT CELA REPRÉSENTE-T-IL ?}

Huit cent mille dollars que nous prêtons annuellement. Donc, nous avons environ huit cent mille, neuf cent mille à l'année. Par exemple, là on vient de m'appeler et la personne voulait cent mille.

L'argent total que les investisseurs nous ont prêté est un million et demi. Et ensuite ce que nous avons prêté nous... Eux, ils nous ont prêté un million et demi de dollars et nous avons prêté presque cinq millions de cela plus ce que nous avons dépensé... C'est un projet très beau parce que tu vois la satisfaction des gens par exemple l'autre jour un jeune homme est venu et il me dit... Sa mère m'avait dit qu'il travaillait maintenant à Wells Fargo, nous lui avions prêté pour qu'il fasse sa procédure de DREAMer ${ }^{11}$ et je l'avais vu cette fois-là et personne ne voulait leur prêter, nous leur avons prêté et ensuite la mère a continué et ensuite le jeune homme m'a dit que 
maintenant il avait un travail à Wells Fargo et qu'il s'était acheté la première voiture de sa vie! Donc l'autre jour ils sont venus et je leur ai dit que maintenant ils pouvaient obtenir un prêt n'importe où, et le jeune homme m'a dit « Non, parce qu'ici on m'a prêté la première fois et c'est donc ici que je reviens! » et il a dit «Et maintenant je veux acheter une maison pour ma mère ». Donc quand tu entends ces histoires que certains ont reçu leur Green Card, que tu as libéré une femme de la violence domestique et qu'on lui a donné son visa $\mathrm{U}^{12}$, c'est très satisfaisant mais, oui, c'est très fastidieux parce que du dois être là tout le temps et ça prend beaucoup de temps du Front parce que bon, le Front aussi requiert beaucoup de temps, être avec les organisations, être avec les communautés, et quand tu es avec les chiffres... Tu ne trouves pas d'équilibre.

\section{MAIS MAINTENANT VOUS AVEZ AUSSI L'APPUI DU FIOB DANS CELA ?}

Eh bien, là où il y a de l'argent, c'est toujours un problème. Parce que comme cela a été créé comme un projet du Front, certains attendaient de savoir comment nous allions répartir les bénéfices. Non, mais il n'y avait pas de bénéfice parce que le projet avait besoin de beaucoup de temps, d'investissement et beaucoup d'argent d'investissement du fait que c'est un système de compte qui se crée, et je crois que nous n'aurons aucun bénéfice avant probablement l'année prochaine, nous commençons à passer le fameux point d'équilibre. Oh enfin! Enfin nous allons pouvoir avoir des salaires décents... Après sept ans! Nous allons enfin pouvoir avoir des salaires décents, nous pourrons enfin avoir... Ce n'est pas qu'ils soient injustes à l'heure actuelle mais un vrai salaire qui montre que réellement le projet a fonctionné parce que pour le moment ce sont des salaires au salaire minimum, parce que le projet ne donne pas pour plus. Non, donc, alors le Front a voulu se séparer du projet, ils ont dit « eh bien non, nous ne voulons plus appuyer le projet parce qu'il n'y a pas de bénéfice, et ça va faire perdre beaucoup de temps, nous n'allons pas participer à ça ». Et donc ils se sont séparés eux-mêmes du projet dans une assemblée. Mais quand quelqu'un est déjà dedans jusqu'au cou, il ne peut pas en sortir comme ça, tu dois aller de l'avant, pas le choix, ou tu laisses tomber, il vaut mieux aller de l'avant avec le projet qu'avoir perdu six ans, cinq ans de ta vie... dans ce projet. C'est vraiment super mais oui, c'est difficile.

\section{c'est votre projet à vous, celui que vous avez créé et que vous dirigez ACTUELLEMENT ?}

Oui c'est un projet que j'ai accepté avec Gaspar quand nous étions les dirigeants principaux et j'ai pris à ma charge ce projet. J'ai pris ce projet à ma charge, obtenir les permis adéquats, maintenant nous avons des avocats. Nous n'aurions jamais pensé avoir des avocats. Nous n'avions jamais pensé que nous aurions une entreprise de comptabilité qui nous fasse la comptabilité... En fait c'est tout une entreprise, nous pensions que c'était très facile, que tu allais prêter l'argent, le récupérer, le mettre à la banque et le prêter de nouveau ! Et en fait il a fallu obtenir des permis, payer des impôts, payer des salaires, donc nous avons tout appris petit à petit aussi, c'est que nous étions tous des activistes, aucun de nous ne se consacrait à cela. Nous avons dû l'apprendre hands-on comme ils disent en anglais. Nous allions demander et oh! Nous devons payer des impôts! Oh, Nous avons besoin de cela !... 


\section{CETTE EXPÉRIENCE VOUS LA TRANSMETTEZ ENSUITE AUX AUTRES MIgRANTS QUI ARRIVENT ICI, POUR MONTER DES ENTREPRISES ?}

Je crois que oui, je crois que... nous aimerions que ces modèles se multiplient, non? Parce que ce modèle, il est très humain. Un exemple... si quelqu'un tombe au travail, nous travaillons avec eux pour qu'ils puissent... ils nous rendent l'argent et ils paient mais d'une manière très humaine, rien à voir avec les corporations que tu dois payer dans les délais sinon ils te punissent. Nous leur parlons, nous travaillons avec eux, en accord avec leurs besoins, et le Front dont ce projet est issu est une organisation de droits humains et nous devons traiter tout le monde humainement et dans le cadre des possibilités, donc qu'ils aient du retard, mais qu'ils paient. Et nous leur faisons comprendre que c'est un petit projet, au moment où ils ne paient pas, eh bien quelqu'un d'autre ne recevra pas de prêt, quelqu'un d'autre ne recevra pas d'aide s'ils ne sont pas ponctuels. Donc ça fait partie de l'organisation.

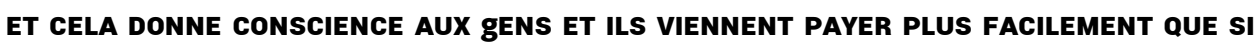 C'ÉTAIT UN AUTRE ORgANISME dE PRÊT ?}

Je crois que certains oui. Parce que certains savent que cela vient du Front et ils disent «En fin de compte, l'argent c'est du capital », ils disent « ils ne vont rien te faire si tu ne paies pas ». Il y en a qui disent « Eh bien, ça vient du FIOB donc il n'y a pas à payer ". Il y en a d'autres qui sont très responsables. Les gens que nous avons rencontrés qui sont les plus responsables sont ceux qui gagnent le salaire minimum ou moins du salaire minimum... Les gens qui ont déjà des papiers ou les gens du mouvement de droits des migrants en lui-même, du mouvement migrant, ils ne paient pas. Ou alors c'est beaucoup de travail pour qu'ils paient. Comme ils connaissent déjà le système, la bureaucratie pour les amener au tribunal et tout cela... certains nous ont dit « Eh bien, je sais que vous ne pouvez rien faire, qu'est-ce qu'ils peuvent me faire? ». Et là on vient d'en amener un au tribunal pour qu'il paie. Parce qu'il est venu avec toute l'intention de ne pas payer, et c'est quelqu'un qui a des papiers, c'est quelqu'un qui fait partie du mouvement des migrants. Il y a eu une organisation à qui nous avons prêté pour qu'elle fasse son projet comme nous, pour une communauté, et en échange elle ne nous a pas remboursés, nous avons dû l'amener devant la cour, nous venons de gagner le procès. Donc, c'est une prise de tête ce projet ! C'est très satisfaisant mais il y a aussi beaucoup de prises de tête. Quand les gens ne te paient pas, tu sais que tu dois l'argent aux investisseurs et donc il faut faire tout ce qui est possible pour le récupérer. Mais tu dois aussi garder à l'esprit les droits humains, ceux des gens, non ? Et voir dans quelles conditions ils vivent, s'ils n'ont pas de travail, s'ils tombent malades, s'ils perdent un proche... Et donc nous prenons tout cela en compte, et donc quand on les emmène au tribunal c'est parce que depuis le début ils sont venus avec l'intention de te voler.

\section{ET gÉnÉRALEMENT, VOS CLIENTS SONT DE OAXACA, COMME VOUS ?}

Nous avons... Ça dépend. Par exemple à Santa Maria tous nos clients sont Mixtèques. À Oxnard, pareil. À Los Angeles nous en avons beaucoup du Guatemala, mais nous en avons aussi beaucoup de Jalisco, de Michoacán, de Zacatecas et beaucoup de Salvadoriens ${ }^{13}$. Oui. Mais très peu de Oaxaqueños. Au Crédit, tous ceux qui travaillent... nous sommes tous Oaxaqueños, et toutes des femmes, il n'y a qu'un seul homme. Celui qui dirige Santa Maria c'est le seul homme du projet. Mais tous les autres sont des femmes. 
Cette organisation du fiob est présente dans d'aUtres parties du mexiQue ou SEULEMENT À OAXACA?

À Oaxaca et à San Quintin, Basse Californie, et à Tijuana. Pour les populations migrantes. Ici nous avons des membres par exemple de Guerrero, de Puebla, mais parce qu'ils sont à San Quintin ou parce qu'ils sont en Californie. Nous n'avons pas de membres à Puebla ou au Guerrero mais nous avons des membres du Guerrero et de Puebla parce qu'ils vivent maintenant à San Quintin ou parce qu'ils vivent maintenant en Californie.

\section{À côté de tout ce travall au Crédit entrepreneur et pour les droits humains, VOUS ÊTES AUSSI INTERPRÈTE N'EST-CE PAS ?}

Ah, oui. D'anglais, d'espagnol, de zapotèque et de conneries ! [Rires]. Oui, moi je suis devenue interprète enfant, quand la communauté par exemple... quand j'ai commencé à apprendre l'anglais, bon c'est clair que je ne parlais pas anglais mais on disait que oui... J'avais dix ans quand je suis arrivée ici et je parlais seulement zapotèque. Avec deux ou trois mots en espagnol... quelque chose de très basique. Et peu à peu j'ai commencé à aller à l'école et j'ai appris à maîtriser l'anglais. Mais une des choses c'est qu'ensuite quelqu'un m'a demandé que je sois son interprète pour passer son examen de coiffure, pour l'État de Californie. Et j'y suis allée, je ne sais pas comment nous avons réussi l'examen parce que.... mais nous l'avons eu. Et maintenant je me rends compte après avoir étudié l'interprétariat et la traduction, j'ai dit « quelle horreur ! ». Qui sait comment nous avons fait mais... J'ai commencé... comment vous dites-vous ? Interprète empirique? [Rires]. Mais moi je suis allée très tard à l'école, aux cours d'interprétariat. Moi j'ai commencé comme ça, de rien, sans parler correctement l'anglais ni l'espagnol. Je ne maîtrisais toujours pas parfaitement le zapotèque en ce temps-là. Mais non. Et après, j'ai commencé à le faire... j'ai commencé à le faire comme une habitude de faire de l'interprétariat. Beaucoup plus tard je suis allée à l'école, et puis, je fais seulement... je ne le fais presque plus... bon Zoogocho ne nécessite presque plus d'interprète maintenant, parce que comme nous sommes arrivés ici il y a longtemps, il y a eu peu de fois où j'ai fait l'interprète pour des gens de Zoogocho, deux ou trois cas pas plus. Et à Los Angeles, eh bien on a chaque jour moins besoin d'interprètes parce que la langue se perd et les Indiens commencent à maîtriser l'espagnol, l'anglais, et donc voilà... Ils n'ont plus besoin d'interprète mais oui, ça s'est fait avec d'autres communautés et ça continue à se faire avec d'autres communautés qui ont besoin d'interprètes. Et après je suis devenue interprète d'espagnol à l'anglais mais dans les mouvements sociaux, les mouvements indiens. Et ensuite quand il y a une conférence ou autre eh bien je travaille comme interprète de l'anglais à l'espagnol.

EN JANVier dernier vous aVez ORganisé un ATELIER AVeC LA LAPD, LA POLICE De LOS ANgELES, SUR CES QUESTIONS DE LANgUES INDIENNES ET D'INTERPRÉtARIAT. CELA FAIT PARTIE DE VOS ACTIVITÉS AU TRAVERS DU FIOB OU L'AVEZ-VOUS FAIT DANS LE CADRE DE VOTRE FONCTION D'INTERPRÈTE?

Cela vient d'un projet du Front, d'éduquer les fonctionnaires sur la migration indienne, pour différents cas qu'il y a eu, les années passées, parce que les gens, et les fonctionnaires et les gens, les Mexicains en général, ne savaient pas qu'il y avait des communautés indiennes. Et ainsi beaucoup ont fini injustement en prison, certains qui... Comment s'appelle cette femme qui a écrit sur ça ? Je vais te le dire pour que tu aies le nom... Cette femme qui s'appelle Lourdes De León ${ }^{14}$ a écrit la vie de cet homme Triqui qui a été mis dans un asile, où ils mettent les gens dont ils pensent qu'ils sont 
mal dans leur tête, qu'ils sont fous. Et lui ils l'ont mis dans une institution mentale parce que personne ne savait qu'il ne parlait pas espagnol. Et ils disaient qu'il faisait des bruits bizarres, et les gens ne savaient pas que c'était une autre langue indienne. Donc, pour toutes ces expériences, le Front a décidé de faire ces ateliers d'éducation pour tous ces... fonctionnaires, pour les hôpitaux, les écoles et tout. Et cela a été un succès. Au niveau de la Californie, cela a été fait pour l'institution fiscale, pour les juges, pour la police de Los Angeles, pour la police de Santa Maria, pour le comté d'Oxnard, ça a été fait à Sacramento... Tout cela sur la migration indienne. C'est quelque chose de vraiment unique qu'a lancé le Front il y de nombreuses années. Le Front a aussi lancé un projet d'interprètes en 1993, ils ont obtenu leur diplôme, un groupe d'interprètes indiens, ils ont presque tous obtenu leur diplôme. Il y avait des Mayas, il y avait des Mixtèques et des Zapotèques, et le Front leur a payé les cours de base, les cours basiques de... de comment faire de l'interprétariat, la confidentialité et l'éthique. Et alors maintenant il y a deux autres organisations qui ont un groupe d'interprètes, d'autres organisations commencent à faire des ateliers de sensibilisation culturelle. C'est quelque chose que le Front a lancé il y a de nombreuses années, et c'est bien qu'ils le fassent parce que le Front n'est pas suffisant pour tout l'État de Californie. Donc nous sommes contents que les gens... comment on dit? Il n'y a pas meilleur compliment que quand les gens refont ce que tu fais, donc c'est très satisfaisant pour nous, que d'autres le fassent, que quelqu'un continue ce que le Front a commencé il y a quinze ou vingt ans.

\section{SELON VOUS, LA SITUATION DES MIgRANTS INDIENS S'EST-ELLe AMÉLIORÉE ICI EN CALIFORNIE AVEC TOUT LE TRAVAIL DU FRONT?}

Eh bien... Je crois que oui ! Il y a une meilleure prise de conscience de la présence des migrants indiens et nous avons obtenu ce que nous n'avons pas obtenu dans notre pays : avoir un interprète... quand tu es dans un tribunal, quand tu es dans un hôpital, et donc, il y a encore beaucoup à faire, mais nous avons déjà quelque chose de super avancé en ce qui concerne le droit d'avoir un interprète face au tribunal, face aux cliniques, face à l'école. Et nous avons fait des campagnes des deux côtés: la communauté qui a droit à un interprète et aussi les fonctionnaires. Ils ont des droits, l'obligation de pourvoir un interprète en langue indienne. Et on travaille là-dessus. Et c'est quelque chose qui n'a pas été obtenu au Mexique, et quand on voit les statistiques de populations indiennes emprisonnées, le fait que ces populations ne bénéficient presque jamais d'un avocat, c'est très triste, et ça continue d'être triste. Mais nous n'avons pas obtenu cela au Mexique. De fait, c'est triste à dire mais nous avons été mieux traités ici qu'au Mexique, et de fait nous faisons le travail que les consulats devraient faire. Le consulat devrait pourvoir les interprètes, le consulat devrait travailler à la défense des droits des communautés indiennes, mais il n'en est pas ainsi. Nous, nous formons les interprètes. Le consulat nous appelle nous pour nous dire «j'ai besoin d'un interprète en telle langue». En fait les bureaux du gouvernement qui sont ici... Oh! C'est ce qui se passe à Santa Maria, «nous avons besoin de l'aide du Front». Mince alors! Donc vous, vous avez un salaire, vous avez des fonds, pourquoi vous venez nous chercher nous? Mais en fait il y a une solidarité avec les compatriotes, il faut le faire mais c'est presque une honte que les consulats au niveau national ne fournissent pas les services adéquats pour les communautés indiennes. Mais par contre ils t'appellent pour danser, ils t'appellent pour faire un évènement d'Indiens, là, avec différentes danses, et des chants, et pour que tu mettes ton huipil et te montrer ainsi exotique, hein ? Mais à l'heure de servir la communauté 
dans un tribunal, avec un avocat, avec tout ça, il n'y a pas de soutien. C'est pour ça que je n'aime pas les consulats.

\section{Y A-T-IL QUelque chose QUe vous voulez RAJOUter À propos du front, de Votre IMPLICATION?}

Eh bien le Front me plaît beaucoup, il est unique, il est très complexe comme ils diraient ici, c'est... Il y a de tout, il y a beaucoup à faire encore mais beaucoup de choses ont aussi été obtenues au travers du Front, comme la reconnaissance des communautés indiennes, dans les tribunaux, dans les écoles, qu'il y ait beaucoup plus d'organisations qui naissent dans la continuité du Front, peut-être par dissidence face au Front, peu importe pourquoi elles sont nées mais elles sont là. Et elles font un très bon travail. Savoir qu'il y a maintenant une organisation triqui qui œuvre à la défense des droits des Triquis dans le nord de la Californie, moi je suis très émue par cela parce que, comme je te dis, le Front ne peut pas couvrir tout l'État, il n'a pas... la capacité de couvrir tous les problèmes au niveau national des communautés indiennes. Donc c'est un grand plaisir de savoir que des organisations naissent et qu'elles suivent les pas du Front, qu'elles suivent le modèle du Front, et pour nous c'est beaucoup parce que oui, nous avons besoin de cette aide. Oui, le Front est le premier né et tout le monde vient chercher le Front pour tout, mais bon, les cas sont si nombreux, que ce soit des abus dans le travail, des abus sexuels, des cas de violation des droits de l'Homme, que c'est impossible de tout couvrir, n'est-ce pas ? La question de San Quintin ${ }^{15}$, le Front a été très actif avec la collecte de fonds pour apporter de l'aide aux travailleurs journaliers qui étaient en grève. Et ça, c'est notre fonction, on les appuie pour diffuser la situation dans tous les médias internationaux et nationaux et j'ai été très heureuse quand cette femme de Nouvelle Zélande l'autre jour me dit «Moi je veux parler avec les gens du groupe qui est allé fermer les bureaux de Driscoll's, la compagnie de fraises de Californie ». Et moi je lui ai dit "Comment tu as entendu parler de ça, toi ?", et elle m'a dit "C'est parce que c'est sorti dans les informations. Ils en ont parlé aux informations de la situation de la fraise à San Quintin, on a vu la situation du... comment ça s'appelle ? du fait qu'ils ont fermé les bureaux de Driscoll's, et moi je veux parler avec ce groupe ». Et je lui ai dit «Hello! » [Rires].

\section{CE SONT LES ACTIONS QUE VOUS AVEZ MENÉES LÀ-BAS À SAN QUINTIN AVEC LE FRONT ?}

Oui, oui. Avec le Front. Donc le Front est célèbre au niveau mondial sur le fait qu'il peut apporter son appui quand il y a quelque chose comme ça, avec nos moyens de communication, avec les alliés et oui, c'est possible : pour que quelqu'un de Nouvelle Zélande demande, c'est quand même super de savoir que la nouvelle arrive jusque làbas, non ? Et de fait ils disent « Eh bien nous les communautés indigènes, nous avons décidé de ne pas acheter de fraises qui viennent des États-Unis ». Donc c'est qu'il y a de la solidarité, tu imagines ?! Donc ce n'est pas quelque chose de tangible, il n'y a aucun moyen de savoir, parce que comment je pouvais le savoir moi qu'ils étaient en train de faire ça en Nouvelle Zélande et en Australie si ces camarades ne me l'avaient pas dit? Mais elles le font! Qu'elles n'achètent pas les fraises des États-Unis, that is pretty cool!

Quand quelqu'un est en grève, il a besoin de beaucoup de soutien parce qu'alors il ne reçoit pas de salaire... Comment tu manges? Comment tu donnes à manger à ta famille ? Donc c'est un acte de solidarité. Ce n'est pas de la pitié mais de la solidarité de dire « Ok, nous avons besoin que tu continues la lutte et nous allons te donner un 
coup de main de cette manière ", et à travers ça faire les dénonciations dans les médias. Je crois que ce n'est pas la première fois que le Front le fait. Le Front l'a fait en 2006 avec la grève des enseignants qu'il y a eu à Oaxaca ${ }^{16}$, en fait il y a eu une solidarité très grande depuis ici. Nous avons envoyé de l'argent mais nous avons aussi fait des dénonciations dans les médias, au travers des réseaux dans les universités, avec les communautés, pour dénoncer ce qu'il se passe, la répression, ce qui était en train de se passer là-bas. Donc c'est un travail que le Front a toujours fait. Le Front a de nombreux fronts: il a cette partie, la sensibilisation culturelle, la partie des microcrédits, la partie des femmes, et la participation politique locale. Parce qu'il ne participe pas seulement au niveau binational mais il est impliqué dans l'élection du maire de Los Angeles, il fait des collectes de fonds pour le maire de Los Angeles quand... ou pour les conseillers municipaux. Et donc c'est un peu... il a différent fronts, le Front. Parce que si nous pouvons soutenir un homme politique, alors nous avons soutenu Eric Garcetti la dernière fois, lors de la dernière élection du maire de Los Angeles; nous avons soutenu Curren D. Price, Marqueece Harris-Dawson ${ }^{17}$, pour les derniers, et d'ailleurs aujourd'hui premier juillet c'est quand officiellement les conseillers municipaux que nous avons soutenus dans leurs campagnes entrent en fonction. Et donc tout cela fait partie de... parce que si tu viens, si nous sommes aux États-Unis, nous voulons garder nos coutumes, nos langues, nos danses traditionnelles, pas les folkloriques mais les traditionnelles, nous devons aussi participer à la politique locale parce que nous vivons ici et que nous avons des enfants ici et donc nous ne pouvons pas être étrangers à la politique locale de la ville. Et le Front est ainsi, de tous les côtés. Ceux de Basse Californie sont impliqués dans la politique locale, ceux de Californie, et bien sûr ceux de Oaxaca sont très impliqués dans la question politique!

\section{NOTES}

1. Les références au FIOB dans la littérature spécialisée sont nombreuses. Pour en savoir plus sur cette organisation, on citera notamment:

Domínguez, R., 2004, «The Fiob experience: Internal crisis and future challenges », in J. Fox et G. Rivera-Salgado (ed.), Indigenous Mexican Migrants in the United States. Center for U.S-Mexican Studies, La Jolla: Center for Comparative Immigration Studies, UCSD , pp. 69-79.

Lestage F., 2002, « Manejar la complejidad del transnacionalismo. A propósito de algunas redes de los migrantes oaxaqueños ", in F. Morin et R. Santana (ed.), Lo transnacional. Instrumento y desafío para los pueblos indigenas, Quito: Abya Yala, pp.169-201.

2. Le huipil, du terme nahuatl huipilli, est le vêtement traditionnel commun porté par les femmes autochtones du centre du Mexique et de l'Amérique centrale.

3. Les remesas sont les envois, le plus souvent d'argent, effectués par les migrants vers leur région d'origine.

4. L'argent des remesas est mis en commun au travers de ces caisses d'épargne. Les fonds ainsi constitués sont collectivement mis à disposition au travers de ces coopératives de micro crédit. En plus de permettre le financement de projets qui ne pourraient voir le jour sans cet usage 
collectifs des remesas, la division entre les bailleurs des profits engendrés par les taux d'intérêt dégage une source de revenus supplémentaire (même si cela reste très modeste).

5. «Paycheck» est un nom communément employé pour parler de petites entreprises dont l'activité consiste à avancer l'équivalent du salaire du demandeur et qui se font rembourser le jour même de la réception du solde.

6. Le test de Papanicolaou, ou frottis vaginal, est un examen servant à détecter des modifications anormales des cellules du col de l'utérus et qui permet ainsi de prévenir le développement du cancer du col de l'utérus.

7. Odilia Romero utilise le terme espagnol de empoderamiento, qui fait directement référence au concept d'empowerment. Pour faciliter la lecture, et en l'absence d'une traduction satisfaisante en français, le terme en espagnol a été à chaque fois remplacé par sa traduction en anglais.

8. Préparation culinaire très populaire au Mexique, à base de galettes de maïs rassises et de sauce au piment, selon les cas de couleur rouge ou verte, d'où la métaphore.

9. Le terme de oriundos se réfère à des individus originaires d'une même communauté ou d'une même région.

10. Gaspar Rivera-Salgado, originaire de la région Mixtèque de l'État de Oaxaca, est sociologue et directeur de projet au sein du Center for Labor Research and Education de UCLA. Il est également consultant indépendant sur les questions de migration transnationale et relations ethniques.

11. Le terme de DREAMer fait référence au DREAM Act (Development, Relief and Education for Alien Minors), le décret de régularisation votée en 2012 en faveur des individus arrivés enfant aux États-Unis et ayant fait des études ou étant engagés dans l'armée.

12. Visa attribué aux victimes d'activités criminelles.

13. L'accès au crédit est ouvert à tous sous couvert de certaines conditions. Pour obtenir un prêt, les clients doivent fournir une pièce d'identité avec photo (permis de conduire, passeport, carte du consulat..), un justificatif de domicile, apporter une preuve qu'ils travaillent (talons des trois derniers chèques de salaire ou lettre de l'employeur), remplir un formulaire et donner les coordonnées de six références personnelles. Les sommes prêtées vont de $\$ 500$ à $\$ 1000$ pour un remboursement entre 3 et 6 mois à un taux d'intérêt de $5 \%$ par mois. Ces montants pouvaient être plus élevés par le passé mais les problèmes de remboursement rencontrés ont amené les membres du Crédit Entrepreneur à revoir leur offre à la baisse.

14. Lourdes de León Pasquel est Professeur de linguistique et d'anthropologie au sein du Centro de Investigaciones y Estudios Superiores en Antropología Social (CIESAS-Sureste) au Mexique. Elle est l'auteure de plusieurs articles sur les langues indiennes du Mexique et l'interprétariat dans les tribunaux aux États-Unis.

15. La situation des travailleurs agricoles dans la Vallée de San Quintin en Basse Californie n'est pas nouvelle car les premiers mouvements de contestation datent de plus de vingt ans. Le dernier soulèvement de mars 2015 a tenté de dénoncer, une fois de plus, les conditions de travail et d'existence dans lesquelles sont contraints de vivre ces travailleurs journaliers.

16. Il s'agit d'un mouvement de grève lancé en mai 2006 par un syndicat d'enseignants qui a rassemblé une grande partie de la population de l'État de Oaxaca contre le gouvernement local. Après six mois d'affrontements avec les forces armées (polices locales, fédérales et l'armée), le mouvement s'est désagrégé sans obtenir la destitution du gouverneur qui était demandée. Pour un récit en français des évènements, voir notamment : Rosen-Cros, 2010, ; Duro Compañer@s ! Oaxaca 2006 : Récits d'une insurrection mexicaine, éd. Tanin-Party.

17. Eric Garcetti, Curren D. Price et Marqueece Harris-Dawson sont tous trois membres du Parti Démocrate. Eric Garcetti est maire de la ville de Los Angeles depuis 2013. Curent D. Price et Marqueece Harris-Dawson sont les représentants au Los Angeles City Council des districts 8 et 9 . Ces districts couvrent notamment les zones de Central L.A., Crenshaw et West Adams dans lesquelles la présence de communautés originaires de Oaxaca est forte. 


\section{AUTEUR}

\section{AMANDINE DEBRUYKER}

Doctorante en Anthropologie sociale et culturelle à Aix-Marseille Université (Idemec) et coordinatrice du Pôle Californie de l'Institut des Amériques. amandinedebruyker@gmail.com / pole.californie@institutdesameriques.fr 